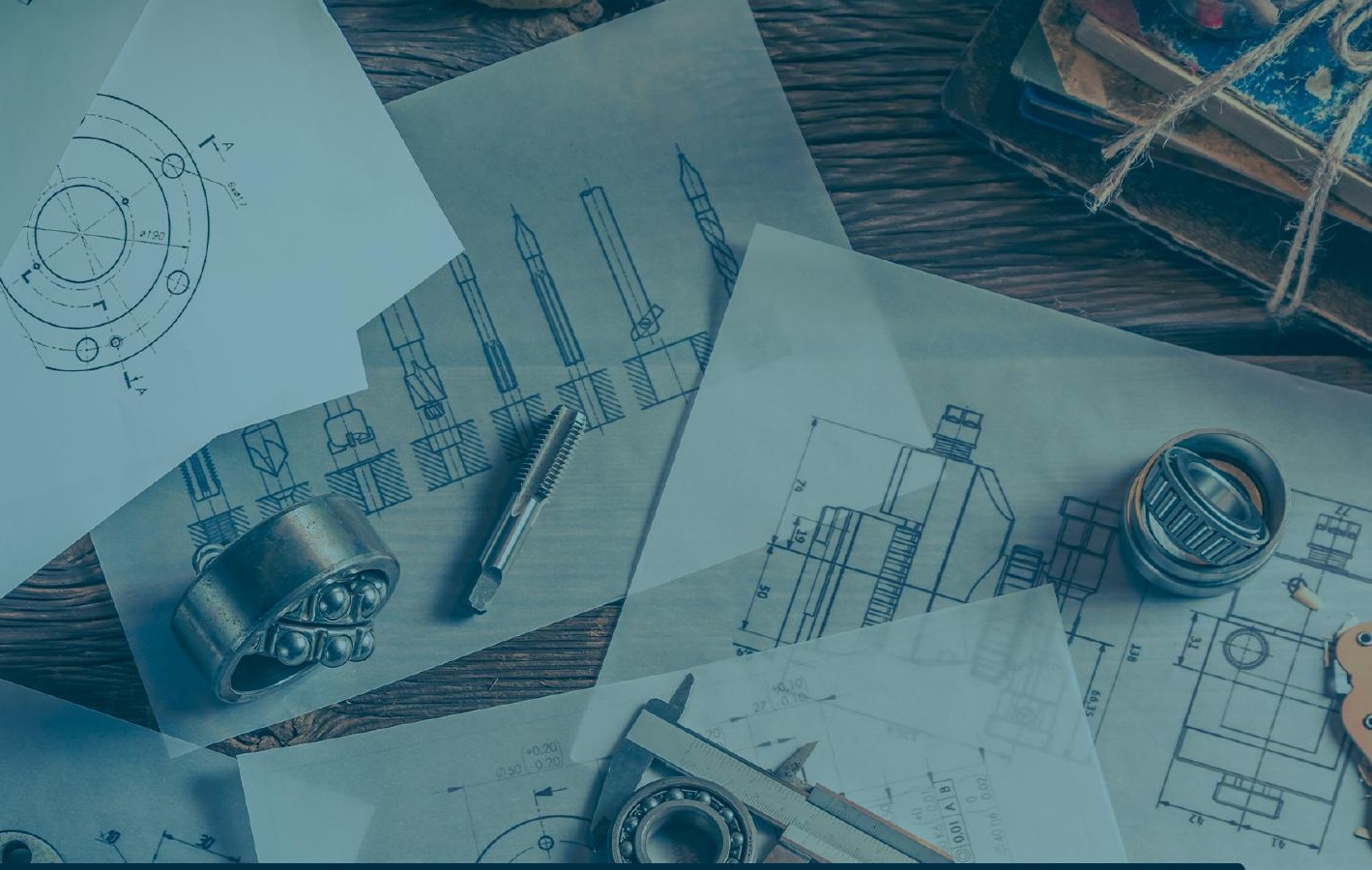

Henrique Ajuz Holzmann

João Dallamuta

(Organizadores)

\title{
A Aplicação do \\ Conhecimento Científico \\ na Engenharia Mecânica
}
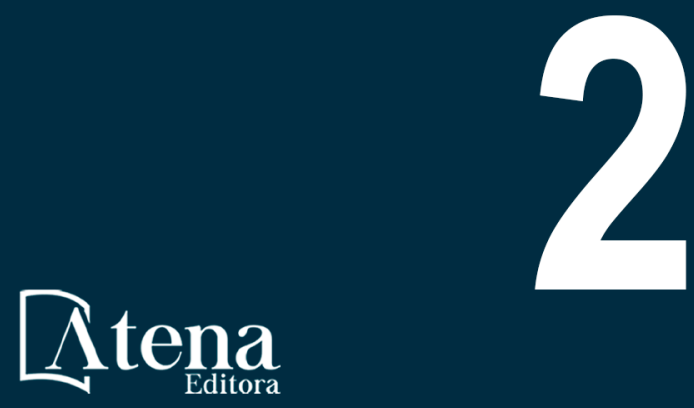

Ano 2020 



\section{Editora Chefe}

Prof $^{\mathrm{a}} \mathrm{Dr}^{\mathrm{a}}$ Antonella Carvalho de Oliveira

Assistentes Editoriais

Natalia Oliveira

Bruno Oliveira

Flávia Roberta Barão

Bibliotecário

Maurício Amormino Júnior

Projeto Gráfico e Diagramação

Natália Sandrini de Azevedo

Camila Alves de Cremo

Karine de Lima Wisniewski

Luiza Alves Batista

Maria Alice Pinheiro

Imagens da Capa

Shutterstock

2020 by Atena Editora

Edição de Arte

Luiza Alves Batista

Copyright (c) Atena Editora

Copyright do Texto (C) 2020 Os autores

Copyright da Edição @ 2020 Atena Editora

Revisão Direitos para esta edição cedidos à Atena

Os Autores Editora pelos autores.

Todo o conteúdo deste livro está licenciado sob uma Licença de Atribuição Creative Commons. Atribuição 4.0 Internacional (CC BY 4.0).

O conteúdo dos artigos e seus dados em sua forma, correção e confiabilidade são de responsabilidade exclusiva dos autores, inclusive não representam necessariamente a posição oficial da Atena Editora. Permitido o download da obra e o compartilhamento desde que sejam atribuídos créditos aos autores, mas sem a possibilidade de alterá-la de nenhuma forma ou utilizá-la para fins comerciais.

A Atena Editora não se responsabiliza por eventuais mudanças ocorridas nos endereços convencionais ou eletrônicos citados nesta obra.

Todos os manuscritos foram previamente submetidos à avaliação cega pelos pares, membros do Conselho Editorial desta Editora, tendo sido aprovados para a publicação.

\section{Conselho Editorial}

\section{Ciências Humanas e Sociais Aplicadas}

Prof. Dr. Álvaro Augusto de Borba Barreto - Universidade Federal de Pelotas

Prof. Dr. Alexandre Jose Schumacher - Instituto Federal de Educação, Ciência e Tecnologia do Paraná

Prof. Dr. Américo Junior Nunes da Silva - Universidade do Estado da Bahia

Prof. Dr. Antonio Carlos Frasson - Universidade Tecnológica Federal do Paraná

Prof. Dr. Antonio Gasparetto Júnior - Instituto Federal do Sudeste de Minas Gerais

Prof. Dr. Antonio Isidro-Filho - Universidade de Brasília

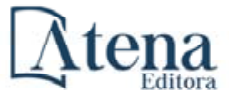


Prof. Dr. Carlos Antonio de Souza Moraes - Universidade Federal Fluminense

Prof $^{a}$ Dr $^{\text {a }}$ Cristina Gaio - Universidade de Lisboa

Prof. Dr. Daniel Richard Sant'Ana - Universidade de Brasília

Prof. Dr. Deyvison de Lima Oliveira - Universidade Federal de Rondônia

Prof $^{a}$ Dr $^{a}$ Dilma Antunes Silva - Universidade Federal de São Paulo

Prof. Dr. Edvaldo Antunes de Farias - Universidade Estácio de Sá

Prof. Dr. Elson Ferreira Costa - Universidade do Estado do Pará

Prof. Dr. Eloi Martins Senhora - Universidade Federal de Roraima

Prof. Dr. Gustavo Henrique Cepolini Ferreira - Universidade Estadual de Montes Claros

Prof $^{a} \mathrm{Dr}^{\mathrm{a}}$ Ivone Goulart Lopes - Istituto Internazionele delle Figlie de Maria Ausiliatrice

Prof. Dr. Jadson Correia de Oliveira - Universidade Católica do Salvador

Prof. Dr. Julio Candido de Meirelles Junior - Universidade Federal Fluminense

Prof $^{a} \mathrm{Dr}^{\mathrm{a}}$ Lina Maria Gonçalves - Universidade Federal do Tocantins

Prof. Dr. Luis Ricardo Fernandes da Costa - Universidade Estadual de Montes Claros

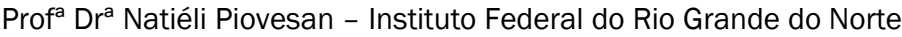

Prof. Dr. Marcelo Pereira da Silva - Pontifícia Universidade Católica de Campinas

Prof $^{a}$ Dr $^{a}$ Maria Luzia da Silva Santana - Universidade Federal de Mato Grosso do Sul

Prof $^{a}$ Dr $^{a}$ Paola Andressa Scortegagna - Universidade Estadual de Ponta Grossa

Prof $^{a}$ Dr $^{a}$ Rita de Cássia da Silva Oliveira - Universidade Estadual de Ponta Grossa

Prof. Dr. Rui Maia Diamantino - Universidade Salvador

Prof. Dr. Urandi João Rodrigues Junior - Universidade Federal do Oeste do Pará

Prof $^{a}$ Dr $^{a}$ Vanessa Bordin Viera - Universidade Federal de Campina Grande

Prof. Dr. William Cleber Domingues Silva - Universidade Federal Rural do Rio de Janeiro

Prof. Dr. Willian Douglas Guilherme - Universidade Federal do Tocantins

\section{Ciências Agrárias e Multidisciplinar}

Prof. Dr. Alexandre Igor Azevedo Pereira - Instituto Federal Goiano

Prof $^{\mathrm{a}} \mathrm{Dr}^{\mathrm{a}}$ Carla Cristina Bauermann Brasil - Universidade Federal de Santa Maria

Prof. Dr. Antonio Pasqualetto - Pontifícia Universidade Católica de Goiás

Prof. Dr. Cleberton Correia Santos - Universidade Federal da Grande Dourados

Prof $^{a}$ Dr $^{a}$ Daiane Garabeli Trojan - Universidade Norte do Paraná

Prof ${ }^{a}$ Dr $^{a}$ Diocléa Almeida Seabra Silva - Universidade Federal Rural da Amazônia

Prof. Dr. Écio Souza Diniz - Universidade Federal de Viçosa

Prof. Dr. Fábio Steiner - Universidade Estadual de Mato Grosso do Sul

Prof. Dr. Fágner Cavalcante Patrocínio dos Santos - Universidade Federal do Ceará

Prof ${ }^{a}$ Dr $^{a}$ Girlene Santos de Souza - Universidade Federal do Recôncavo da Bahia

Prof. Dr. Jael Soares Batista - Universidade Federal Rural do Semi-Árido

Prof. Dr. Júlio César Ribeiro - Universidade Federal Rural do Rio de Janeiro

Prof $^{a}$ Dr $^{a}$ Lina Raquel Santos Araújo - Universidade Estadual do Ceará

Prof. Dr. Pedro Manuel Villa - Universidade Federal de Viçosa

Prof $^{a}$ Dr $^{a}$ Raissa Rachel Salustriano da Silva Matos - Universidade Federal do Maranhão

Prof. Dr. Ronilson Freitas de Souza - Universidade do Estado do Pará

Prof $^{a}$ Dr $^{a}$ Talita de Santos Matos - Universidade Federal Rural do Rio de Janeiro

Prof. Dr. Tiago da Silva Teófilo - Universidade Federal Rural do Semi-Árido

Prof. Dr. Valdemar Antonio Paffaro Junior - Universidade Federal de Alfenas

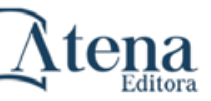

Ano 2020 


\section{Ciências Biológicas e da Saúde}

Prof. Dr. André Ribeiro da Silva - Universidade de Brasília

Prof $^{a}$ Dr $^{a}$ Anelise Levay Murari - Universidade Federal de Pelotas

Prof. Dr. Benedito Rodrigues da Silva Neto - Universidade Federal de Goiás

Prof $^{a}$ Dr $^{a}$ Débora Luana Ribeiro Pessoa - Universidade Federal do Maranhão

Prof. Dr. Douglas Siqueira de Almeida Chaves -Universidade Federal Rural do Rio de Janeiro

Prof. Dr. Edson da Silva - Universidade Federal dos Vales do Jequitinhonha e Mucuri

Prof $^{a}$ Dr $^{a}$ Eleuza Rodrigues Machado - Faculdade Anhanguera de Brasília

Prof $^{\mathrm{a}} \mathrm{Dr}^{\mathrm{a}}$ Elane Schwinden Prudêncio - Universidade Federal de Santa Catarina

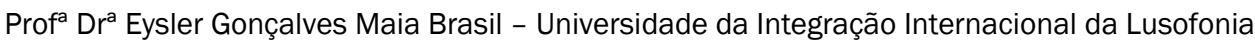
Afro-Brasileira

Prof. Dr. Ferlando Lima Santos - Universidade Federal do Recôncavo da Bahia

Prof $^{a}$ Dr $^{\text {a }}$ Gabriela Vieira do Amaral - Universidade de Vassouras

Prof. Dr. Gianfábio Pimentel Franco - Universidade Federal de Santa Maria

Prof. Dr. Helio Franklin Rodrigues de Almeida - Universidade Federal de Rondônia

Prof $^{a}$ Dr $^{a}$ lara Lúcia Tescarollo - Universidade São Francisco

Prof. Dr. Igor Luiz Vieira de Lima Santos - Universidade Federal de Campina Grande

Prof. Dr. Jefferson Thiago Souza - Universidade Estadual do Ceará

Prof. Dr. Jesus Rodrigues Lemos - Universidade Federal do Piauí

Prof. Dr. Jônatas de França Barros - Universidade Federal do Rio Grande do Norte

Prof. Dr. José Max Barbosa de Oliveira Junior - Universidade Federal do Oeste do Pará

Prof. Dr. Luís Paulo Souza e Souza - Universidade Federal do Amazonas

Prof $^{a}$ Dr $^{a}$ Magnólia de Araújo Campos - Universidade Federal de Campina Grande

Prof. Dr. Marcus Fernando da Silva Praxedes - Universidade Federal do Recôncavo da Bahia

Prof $^{\mathrm{a}} \mathrm{Dr}^{\mathrm{a}}$ Mylena Andréa Oliveira Torres - Universidade Ceuma

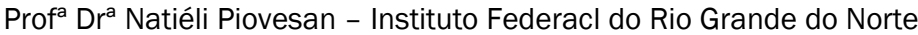

Prof. Dr. Paulo Inada - Universidade Estadual de Maringá

Prof. Dr. Rafael Henrique Silva - Hospital Universitário da Universidade Federal da Grande Dourados

Prof $^{a}$ Dr $^{\mathrm{a}}$ Regiane Luz Carvalho - Centro Universitário das Faculdades Associadas de Ensino

Prof $^{\mathrm{a}} \mathrm{Dr}^{\mathrm{a}}$ Renata Mendes de Freitas - Universidade Federal de Juiz de Fora

Prof $^{\mathrm{a}} \mathrm{Dr}^{\mathrm{a}}$ Vanessa Lima Gonçalves - Universidade Estadual de Ponta Grossa

Prof $^{a}$ Dr $^{a}$ Vanessa Bordin Viera - Universidade Federal de Campina Grande

\section{Ciências Exatas e da Terra e Engenharias}

Prof. Dr. Adélio Alcino Sampaio Castro Machado - Universidade do Porto

Prof. Dr. Alexandre Leite dos Santos Silva - Universidade Federal do Piauí

Prof. Dr. Carlos Eduardo Sanches de Andrade - Universidade Federal de Goiás

Prof ${ }^{a}$ Dr $^{a}$ Carmen Lúcia Voigt - Universidade Norte do Paraná

Prof. Dr. Douglas Gonçalves da Silva - Universidade Estadual do Sudoeste da Bahia

Prof. Dr. Eloi Rufato Junior - Universidade Tecnológica Federal do Paraná

Prof. Dr. Fabrício Menezes Ramos - Instituto Federal do Pará

Prof $^{a}$ Dra. Jéssica Verger Nardeli - Universidade Estadual Paulista Júlio de Mesquita Filho

Prof. Dr. Juliano Carlo Rufino de Freitas - Universidade Federal de Campina Grande

Prof $^{a}$ Dr $^{a}$ Luciana do Nascimento Mendes - Instituto Federal de Educação, Ciência e Tecnologia do Rio Grande do Norte

Prof. Dr. Marcelo Marques - Universidade Estadual de Maringá

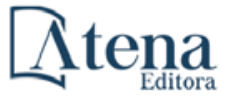




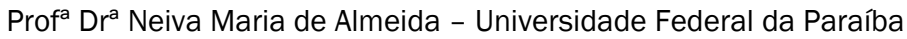

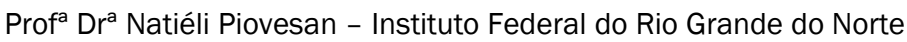

Prof. Dr. Takeshy Tachizawa - Faculdade de Campo Limpo Paulista

\section{Linguística, Letras e Artes}

Prof $^{a}$ Dr $^{a}$ Adriana Demite Stephani - Universidade Federal do Tocantins

Prof $^{a}$ Dr $^{a}$ Angeli Rose do Nascimento - Universidade Federal do Estado do Rio de Janeiro

Prof $^{a}$ Dr $^{a}$ Carolina Fernandes da Silva Mandaji - Universidade Tecnológica Federal do Paraná

Prof $^{a}$ Dr $^{\text {a }}$ Denise Rocha - Universidade Federal do Ceará

Prof. Dr. Fabiano Tadeu Grazioli - Universidade Regional Integrada do Alto Uruguai e das Missões

Prof. Dr. Gilmei Fleck - Universidade Estadual do Oeste do Paraná

Prof $^{a}$ Dr $^{a}$ Keyla Christina Almeida Portela - Instituto Federal de Educação, Ciência e Tecnologia do Paraná

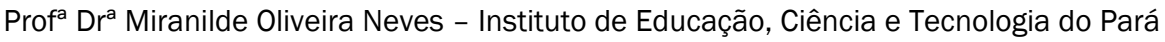

Prof $^{a}$ Dr $^{a}$ Sandra Regina Gardacho Pietrobon - Universidade Estadual do Centro-Oeste

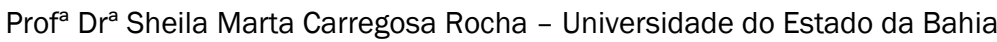

\section{Conselho Técnico Científico}

Prof. Me. Abrãao Carvalho Nogueira - Universidade Federal do Espírito Santo

Prof. Me. Adalberto Zorzo - Centro Estadual de Educação Tecnológica Paula Souza

Prof. Me. Adalto Moreira Braz - Universidade Federal de Goiás

Prof. Dr. Adaylson Wagner Sousa de Vasconcelos - Ordem dos Advogados do Brasil/Seccional Paraíba

Prof. Dr. Adilson Tadeu Basquerote Silva - Universidade para o Desenvolvimento do Alto Vale do Itajaí

Prof. Me. Alexsandro Teixeira Ribeiro - Centro Universitário Internacional

Prof. Me. André Flávio Gonçalves Silva - Universidade Federal do Maranhão

Prof $^{a}$ Ma. Anne Karynne da Silva Barbosa - Universidade Federal do Maranhão

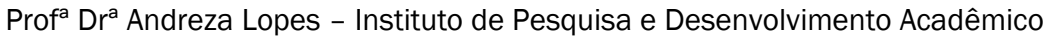

Prof $^{a}$ Dr $^{a}$ Andrezza Miguel da Silva - Faculdade da Amazônia

Prof. Dr. Antonio Hot Pereira de Faria - Polícia Militar de Minas Gerais

Prof. Me. Armando Dias Duarte - Universidade Federal de Pernambuco

Prof $^{a}$ Ma. Bianca Camargo Martins - UniCesumar

Prof $^{a}$ Ma. Carolina Shimomura Nanya - Universidade Federal de São Carlos

Prof. Me. Carlos Antônio dos Santos - Universidade Federal Rural do Rio de Janeiro

Prof. Ma. Cláudia de Araújo Marques - Faculdade de Música do Espírito Santo

Prof $^{a}$ Dr $^{a}$ Cláudia Taís Siqueira Cagliari - Centro Universitário Dinâmica das Cataratas

Prof. Me. Clécio Danilo Dias da Silva - Universidade Federal do Rio Grande do Norte

Prof. Me. Daniel da Silva Miranda - Universidade Federal do Pará

Prof $^{\mathrm{a}}$ Ma. Daniela da Silva Rodrigues - Universidade de Brasília

Prof $^{a}$ Ma. Daniela Remião de Macedo - Universidade de Lisboa

Prof ${ }^{a}$ Ma. Dayane de Melo Barros - Universidade Federal de Pernambuco

Prof. Me. Douglas Santos Mezacas - Universidade Estadual de Goiás

Prof. Me. Edevaldo de Castro Monteiro - Embrapa Agrobiologia

Prof. Me. Eduardo Gomes de Oliveira - Faculdades Unificadas Doctum de Cataguases

Prof. Me. Eduardo Henrique Ferreira - Faculdade Pitágoras de Londrina

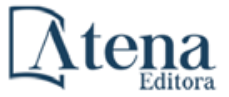


Prof. Dr. Edwaldo Costa - Marinha do Brasil

Prof. Me. Eliel Constantino da Silva - Universidade Estadual Paulista Júlio de Mesquita

Prof. Me. Ernane Rosa Martins - Instituto Federal de Educação, Ciência e Tecnologia de Goiás

Prof. Me. Euvaldo de Sousa Costa Junior - Prefeitura Municipal de São João do Piauí

Prof $^{a}$ Ma. Fabiana Coelho Couto Rocha Corrêa - Centro Universitário Estácio Juiz de Fora

Prof. Dr. Fabiano Lemos Pereira - Prefeitura Municipal de Macaé

Prof. Me. Felipe da Costa Negrão - Universidade Federal do Amazonas

Prof $^{a}$ Dr $^{a}$ Germana Ponce de Leon Ramírez - Centro Universitário Adventista de São Paulo

Prof. Me. Gevair Campos - Instituto Mineiro de Agropecuária

Prof. Dr. Guilherme Renato Gomes - Universidade Norte do Paraná

Prof. Me. Gustavo Krahl - Universidade do Oeste de Santa Catarina

Prof. Me. Helton Rangel Coutinho Junior - Tribunal de Justiça do Estado do Rio de Janeiro

Prof $^{\mathrm{a}} \mathrm{Ma}$. Isabelle Cerqueira Sousa - Universidade de Fortaleza

Prof $^{a}$ Ma. Jaqueline Oliveira Rezende - Universidade Federal de Uberlândia

Prof. Me. Javier Antonio Albornoz - University of Miami and Miami Dade College

Prof. Me. Jhonatan da Silva Lima - Universidade Federal do Pará

Prof. Dr. José Carlos da Silva Mendes - Instituto de Psicologia Cognitiva, Desenvolvimento Humano e Social

Prof. Me. Jose Elyton Batista dos Santos - Universidade Federal de Sergipe

Prof. Me. José Luiz Leonardo de Araujo Pimenta - Instituto Nacional de Investigación Agropecuaria Uruguay

Prof. Me. José Messias Ribeiro Júnior - Instituto Federal de Educação Tecnológica de Pernambuco

Prof $^{a}$ Dr $^{\mathrm{a}}$ Juliana Santana de Curcio - Universidade Federal de Goiás

Prof ${ }^{a}$ Ma. Juliana Thaisa Rodrigues Pacheco - Universidade Estadual de Ponta Grossa

Prof $^{a}$ Dr $^{a}$ Kamilly Souza do Vale - Núcleo de Pesquisas Fenomenológicas/UFPA

Prof. Dr. Kárpio Márcio de Siqueira - Universidade do Estado da Bahia

Prof $^{a}$ Dr $^{a}$ Karina de Araújo Dias - Prefeitura Municipal de Florianópolis

Prof. Dr. Lázaro Castro Silva Nascimento - Laboratório de Fenomenologia \& Subjetividade/UFPR

Prof. Me. Leonardo Tullio - Universidade Estadual de Ponta Grossa

Prof ${ }^{a}$ Ma. Lilian Coelho de Freitas - Instituto Federal do Pará

Prof ${ }^{a}$ Ma. Liliani Aparecida Sereno Fontes de Medeiros - Consórcio CEDERJ

Prof $^{a}$ Dr $^{a}$ Lívia do Carmo Silva - Universidade Federal de Goiás

Prof. Dr. Lucio Marques Vieira Souza - Secretaria de Estado da Educação, do Esporte e da Cultura de Sergipe

Prof. Me. Luis Henrique Almeida Castro - Universidade Federal da Grande Dourados

Prof. Dr. Luan Vinicius Bernardelli - Universidade Estadual do Paraná

Prof. Dr. Michel da Costa - Universidade Metropolitana de Santos

Prof. Dr. Marcelo Máximo Purificação - Fundação Integrada Municipal de Ensino Superior

Prof. Me. Marcos Aurelio Alves e Silva - Instituto Federal de Educação, Ciência e Tecnologia de São Paulo

Prof ${ }^{a}$ Ma. Maria Elanny Damasceno Silva - Universidade Federal do Ceará

Prof ${ }^{a}$ Ma. Marileila Marques Toledo - Universidade Federal dos Vales do Jequitinhonha e Mucuri

Prof. Me. Ricardo Sérgio da Silva - Universidade Federal de Pernambuco

Prof ${ }^{a}$ Ma. Renata Luciane Polsaque Young Blood - UniSecal

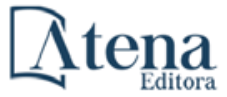


Prof. Me. Robson Lucas Soares da Silva - Universidade Federal da Paraíba

Prof. Me. Sebastião André Barbosa Junior - Universidade Federal Rural de Pernambuco

Prof $^{a}$ Ma. Silene Ribeiro Miranda Barbosa - Consultoria Brasileira de Ensino, Pesquisa e Extensão

Prof $^{a}$ Ma. Solange Aparecida de Souza Monteiro - Instituto Federal de São Paulo

Prof. Me. Tallys Newton Fernandes de Matos - Faculdade Regional Jaguaribana

Prof $^{a}$ Ma. Thatianny Jasmine Castro Martins de Carvalho - Universidade Federal do Piauí

Prof. Me. Tiago Silvio Dedoné - Colégio ECEL Positivo

Prof. Dr. Welleson Feitosa Gazel - Universidade Paulista 
Editora Chefe: Prof $^{\mathrm{a}} \mathrm{Dr}^{\mathrm{a}}$ Antonella Carvalho de Oliveira

Bibliotecário Maurício Amormino Júnior

Diagramação: Luiza Alves Batista

Edição de Arte: Luiza Alves Batista

Revisão: Os Autores

Organizadores: Henrique Ajuz Holzmann

João Dallamuta

Dados Internacionais de Catalogação na Publicação (CIP) (eDOC BRASIL, Belo Horizonte/MG)

A639 A aplicação do conhecimento científico na engenharia mecânica 2 [recurso eletrônico] / Organizadores Henrique Ajuz Holzmann, João Dallamuta. - Ponta Grossa, PR: Atena, 2020.

Formato: PDF

Requisitos de sistema: Adobe Acrobat Reader Modo de acesso: World Wide Web ISBN 978-65-5706-376-7

DOI 10.22533/at.ed.767201109

1. Automação industrial. 2. Engenharia mecânica Pesquisa - Brasil. 3. Produtividade industrial. 4. Tecnologia. I. Holzmann, Henrique Ajuz. II. Dallamuta, João.

Elaborado por Maurício Amormino Júnior - CRB6/2422

Atena Editora

Ponta Grossa - Paraná - Brasil Telefone: +55 (42) 3323-5493 www.atenaeditora.com.br contato@atenaeditora.com.br 


\section{APRESENTAÇÃO}

A Engenharia Mecânica pode ser definida como o ramo da engenharia que aplica os princípios de física e ciência dos materiais para a concepção, análise, fabricação e manutenção de sistemas mecânicos. Nos dias atuais a busca pela redução de custos, aliado a qualidade final dos produtos é um marco na sobrevivência das empresas. Nesta obra é conciliada duas atividades essenciais a um engenheiro mecânico: Projetos e Simulação.

A área de projetos, simulação bem como o desenvolvimento de novo materiais vem ganhando amplo destaque, pois através de simulações pode-se otimizar os projetos realizados, reduzindo o tempo de execução, a utilização de materiais e os custos finais.

Outra área de grande importância é o estudo das naturezas térmicas, pois devido a mudanças significativas no meio ambiente, gradientes cada vez maiores de amplitude térmica vêm sendo registrados. Estes afetem diretamente a processos, previsões de para projetos e ainda aos custos finais de produtos.

Dessa forma, são apresentados trabalhos teóricos e resultados práticos de diferentes formas de aplicação e abordagens nos projetos dentro da grande área das engenharias. Trabalhos envolvendo simulações devido a inserção de novos softwares dedicados a áreas específicas, auxiliando o projetista em suas funções. Sabe-los utilizar de uma maneira eficaz e eficiente é um dos desafios dos novos engenheiros.

Neste livro são apresentados vários trabalhos, alguns com resultados práticos, sobre simulações em vários campos da engenharia industrial, elementos de maquinas e projetos de bancadas práticas.

Um compendio de temas e abordagens que constituem a base de conhecimento de profissionais que se dedicam a projetar e fabricar sistemas mecânicos e industriais.

De abordagem objetiva, a obra se mostra de grande relevância para graduandos, alunos de pós-graduação, docentes e profissionais, apresentando temáticas e metodologias diversificadas, em situações reais.

Boa leitura!

Henrique Ajuz Holzmann

João Dallamuta 


\section{SUMÁRIO}

CAPÍTULO 1

AVALIAÇÃO DA APLICABILIDADE DE EQUAÇÃO TEÓRICA NA PREDIÇÃO DE RUGOSIDADE DO AÇO ABNT 1045 SUBMETIDO AO PROCESSO DE RETIFICAÇÃO

Mayara Fernanda Pereira

Bruno Souza Abrão

Rosemar Batista da Silva

DOI 10.22533/at.ed.7672011091

CAPÍTULO 2

AVALIAÇÃO DA INTEGRIDADE SUPERFICIAL DO AÇO N2711 APÓS RETIFICAÇÃO COM REBOLO DE ÓXIDO DE ALUMÍNIO

Henrique Butzlaff Hübner

Rosemar Batista da Silva

Marcus Antônio Viana Duarte

Eduardo Carlos Bianchi

DOI 10.22533/at.ed.7672011092

CAPÍTULO 3. 15

AVALIAÇÃO DA RUGOSIDADE DO INCONEL 718 APÓS RETIFICAÇÃO COM REBOLOS DE DIFERENTES ABRASIVOS CONVENCIONAIS

Rodrigo de Souza Ruzzi

Raphael Lima de Paiva

Otávio de Souza Ruzzi

Rosemar Batista da Silva

DOI 10.22533/at.ed.7672011093

CAPÍTULO 4 22

AVALIAÇÃO DA RUGOSIDADE GERADA PELO PROCESSO DE JATEAMENTO EM DIFERENTES DISTÂNCIAS DO BOCAL À PEÇA

Henrique Butzlaff Hübner

Antonio Favero Filho

Freddy Alejandro Portillo Morales

Mayara Fernanda Pereira

Rosemar Batista da Silva

DOI 10.22533/at.ed.7672011094

CAPÍTULO 5. 31

ENSAIO BALÍSTICO DE LAMINADOS DE AÇO PARA FUTURA APLICAÇÃO EM BLINDAGEM ADD-ON ESPAÇADA

João Pedro Inácio Varela

Ednaldo Gomes da Rocha Júnior

Wanderley Ferreira de Amorim Júnior

DOI 10.22533/at.ed.7672011095

CAPÍTULO 6. 47

CARACTERIZAÇÃO MECÂNICA DE FILMES HÍBRIDOS BIODEGRADÁVEIS 
COMPOSTOS POR FÉCULA DE BATATA (FB), GELATINA BOVINA (GB) E QUITOSANA (QT)

Francielle Cristine Pereira Gonçalves

Kristy Emanuel Silva Fontes

Elano Costa Silva

Arthur Domingos Mesquita De Morais

Dyana Alves De Oliveira

Théo Martins De Alencar Paiva

Ricardo Alan Da Silva Vieira

Manoel Quirino da Silva Júnior

Francisco Leonardo Gomes de Menezes

DOI 10.22533/at.ed.7672011096

CAPÍTULO 7

REFLECTIVITY BEHAVIOR IN X-BAND OF MICROWAVE ABSORBERS BASED ON CU SUBSTITUTED NIZN SPINEL NANOCRYSTALLINE FERRITE

Valdirene Aparecida da Silva

Evandro Luis Nohara

Mirabel Cerqueira Rezende

DOI 10.22533/at.ed.7672011097

CAPÍTULO 8 71

DESENVOLVIMENTO DE PRÓTESE PERSONALIZADA DA ATM ATRAVÉS DA GERAÇÃO DE IMAGEM 3D BIOMECÂNICA A PARTIR DE TOMOGRAFIA

Rafael Ferreira Gregolin

Cecília Amélia de Carvalho Zavaglia

Ruís Camargo Tokimatsu

João Antônio Pereira

Bruna Beatriz de Paiva

DOI 10.22533/at.ed.7672011098

CAPÍTULO 9

PROJETO ROBUSTO DE CIRCUITO SHUNT RESSONANTE PARA ATENUAÇÃO PASSIVA DE VIBRAÇÕES EM VIGA COMPÓSITA

Lorrane Pereira Ribeiro

Antônio Marcos Gonçalves de Lima

DOI 10.22533/at.ed.7672011099

CAPÍTULO 10.

ESTUDO COMPARATIVO DE DESEMPENHO ENTRE GLP E GNV EM GERADOR À COMBUSTÃO

Paulo Roberto Hahn

Jorge Luis Plácido de Borba

Marco Antônio Frölich

Moisés de Mattos Dias

Elienai Josias Brum Dutra

Monir Göethel Borba

Patrice Monteiro de Aquim 
Eduardo Luis Schneider

José Lesina Cezar

Lirio Schaeffer

DOI 10.22533/at.ed.76720110910

CAPÍTULO 11 104

MODELAGEM DE ESCOAMENTOS GÁS-LÍQUIDO INTERMITENTES: CORRELAÇÕES PARA O COEFICIENTE DE TRANSFERÊNCIA DE CALOR

Lucas Avosani

Luiz Eduardo Melo Lima

DOI 10.22533/at.ed.76720110911

CAPÍTULO 12 117

RADIAÇÃO TÉRMICA EM SUPERFÍCIES SELETIVAS

Gustavo César Pamplona de Sousa

Raimundo Nonato Calazans Duarte

Wanderley Ferreira de Amorim Júnior

Kelly Cristiane Gomes da Silva

DOI 10.22533/at.ed.76720110912

CAPÍTULO 13 128

RESOLUÇÃO DE PROBLEMAS DE CONTROLE ÓTIMO UTILIZANDO ALGORITMOS GENÉTICOS

Arthur Henrique lasbeck

Fran Sérgio Lobato

DOI 10.22533/at.ed.76720110913

CAPÍTULO 14

CONSTRUÇÃO DO TEODOLITO CASEIRO:UMA ALTERNATIVA BARATA E EFICIENTE

Ronis Cley Fontes da Silva

Lourivan Carneiro de Souza

DOI 10.22533/at.ed.76720110914

CAPÍTULO 15 151

ANÁLISE DA POTÊNCIA ELÉTRICA NA RETIFICAÇÃO DO AÇO ENDURECIDO SAE 52100 EM DIFERENTES CONDIÇÕES DE CORTE

Raphael Lima de Paiva

Rodrigo de Souza Ruzzi

Otávio de Souza Ruzzi

Rosemar Batista da Silva

DOI 10.22533/at.ed.76720110915

SOBRE OS ORGANIZADORES

ÍNDICE REMISSIVO 


\section{CAPÍTULO 10}

\section{ESTUDO COMPARATIVO DE DESEMPENHO ENTRE GLP E GNV EM GERADOR À COMBUSTÃO}

Data de aceite: 01/09/2020

Data de submissão: 07/07/2020

Paulo Roberto Hahn

Universidade Feevale

Novo Hamburgo - Rio Grande do Sul

Jorge Luis Plácido de Borba

Universidade Feevale

Novo Hamburgo - Rio Grande do Sul http://lattes.cnpq.br/2128161466465457

\section{Marco Antônio Frölich}

Universidade Feevale

Novo Hamburgo - Rio Grande do Sul

Moisés de Mattos Dias

Universidade Feevale

Novo Hamburgo - Rio Grande do Sul http://lattes.cnpq.br/4783579164324276

\section{Elienai Josias Brum Dutra}

Universidade Feevale

Novo Hamburgo - Rio Grande do Sul http://lattes.cnpq.br/7178930218587378

Monir Göethel Borba

Universidade Federal do Rio Grande do Sul

Porto Alegre - Rio Grande do Sul http://lattes.cnpq.br/3052881710498127

\section{Patrice Monteiro de Aquim}

Universidade Feevale

Novo Hamburgo - Rio Grande do Sul http://lattes.cnpq.br/7098931445685566

\section{Eduardo Luis Schneider}

Universidade Federal do Rio Grande do Sul Porto Alegre - Rio Grande do Sul http://lattes.cnpq.br/4086393631403899
José Lesina Cezar

Universidade Luterana do Brasil Canoas - Rio Grande do Sul http://lattes.cnpq.br/3060084863419387

Lirio Schaeffer Universidade Federal do Rio Grande do Sul Porto Alegre - Rio Grande do Sul http://lattes.cnpq.br/1093242836059112

RESUMO: Geralmente os grupo motor-gerador são movidos a óleo diesel, por ter um custo menor em relação à gasolina, etanol e querosene. $O$ óleo diesel e as novas tecnologias usadas nos grupos geradores têm motivado debates no Brasil, em função da importância do combustível para o desenvolvimento de áreas de Geração de energia, economia, transporte, saúde e meio ambiente. No país já existem inúmeras pesquisas para aprimorar sua qualidade e diminuir o impacto ambiental causado pelas suas emissões. Aanálise técnica para o desenvolvimento de geração de energia pela combustão a gás será desenvolvida através do levantamento da disponibilidade física e compatibilidade do sistema elétrico existente. Um estudo de viabilidade técnica e econômica com fundamentações sólidas tem como objetivo eliminar as dúvidas e paradigmas da utilização de fontes alternativas para a geração de energia elétrica e mostrar, através de comparações, que o sistema estudado é uma opção de geração de energia elétrica econômica no horário de ponta e que pode ser utilizado no fornecimento emergencial ou de segurança. A eficiência energética e a possibilidade de aproveitamento 
de outras fontes de energias renováveis são assuntos muito discutidos nos últimos tempos. Além das descobertas de gás natural, fonte valiosa na produção de energia da Bacia de Santos e outros pontos na camada de pré-sal. Este artigo trata da análise da eficiência, potencial e custo benefício da utilização da geração de energia em sistema GMG a partir da combustão de gás liquefeito de petróleo e gás natural veicular. Os objetivos principais foram abordar e comparar parâmetros de testes realizados em campo em GMG portátil pertencente ao projeto de energias alternativas da Universidade Feevale. Constatou-se diferenças entre o preço $/ \mathrm{m}^{3}$ e grande diferença entre as relações massa-volume-pressão entre os dois gases. Como resultado a utilização do GNV apresentou menor custo.

PALAVRAS-CHAVE: Grupo motor-gerador, gás liquefeito de petróleo, gás natural veicular, energia renovável.

\section{COMPARATIVE STUDY OF PERFORMANCE BETWEEN LPG AND CNG IN COMBUSTION GENERATOR}

ABSTRACT: Generally, the motor-generator groups are powered by diesel fuel, as they have a lower cost in relation to gasoline, ethanol and kerosene. Diesel and new technologies used in generator sets have starts debates in Brazil, due to the importance of fuel for the development of areas of energy generation, economy, transport, health and the environment. There are already numerous researches in the country to improve its quality and reduce the environmental impact caused by its emissions. The technical analysis for the development of power generation by gas combustion will be developed by surveying the physical availability and compatibility of the existing electrical system. A study of technical and economic feasibility with solid foundations aims to eliminate doubts and paradigms in the use of alternative sources for the generation of electric energy and to show, through comparisons, that the studied system is an option of economic electric power generation in the peak hours and can be used for emergency or security supplies. Energy efficiency and the possibility of using other sources of renewable energy are subjects that have been much discussed recently. In addition to the discoveries of natural gas, a valuable source of energy production in the Santos Basin and other points in the pre-salt layer. This paper aims the analysis of efficiency, potential and cost benefit of using energy generation in a motor-generator system from the combustion of liquefied petroleum gas and natural gas. The main objectives were to approach and compare parameters of tests carried out in the field on portable motor-generator belonging to the Feevale University alternative energy project. There were differences between the price/ $\mathrm{m}^{3}$ and a large difference between the mass-volume-pressure relationships between the two gases. As a result, the use of natural gas was less expensive.

KEYWORDS: Motor-generator group, liquefied petroleum gas, natural gas, renewable energy.

\section{I INTRODUÇÃO}

\subsection{Gerador a biometano em sistema híbrido}

A Universidade Feevale, com o financiamento da Secretaria de Ciência e Tecnologia do Estado do Rio Grande do Sul e em parceria com JSA Engenharia, empresa do ramo de projetos de instalações elétricas, desenvolveu um sistema híbrido para geração de energia 
a partir de fontes renováveis (solar, eólica e biomassa). Assim, para a geração de energia a partir de biomassa, foram estudados alguns gases obtidos a partir de material orgânico, como o biometano e seu desempenho em geradores à combustão, também conhecidos como grupo motor-gerador. Neste sentido foi adquirido um gerador a gás alimentado a partir de gás líquido de petróleo (GLP), contudo para a aplicação em energia renováveis, este foi adaptado para gás natural veicular (GNV), o qual deve possuir a mesma formulação do biometano, quando este é purificado. Com o objetivo de verificar o desempenho deste gerador a gás a partir do biometano, foram realizados testes comparativos entre estes dois gases, a partir de cargas resistivas e medições de consumo e potência gerada.

\subsection{Grupo motor-gerador}

Grupo motor-gerador ou grupos geradores são utilizados como fonte principal ou como fonte auxiliar, para suprir a necessidade de energia de forma confiável em empreendimentos de todo e qualquer porte, para quaisquer aplicações, como indústrias, supermercados, shopping centers, hospitais, edifícios residenciais e comerciais, hotéis e outros. Os grupos geradores são constituídos por um gerador, acionado por motor de combustão, sendo este alimentado por combustível (óleo diesel, gás natural, biogás e outros) (Stemac grupos geradores).

Graças aos avanços da tecnologia, os geradores a gás estão crescendo em popularidade para aplicações maiores. Avanços importantes incluem otimização da velocidade de aproximação do motor, integração em paralelo e bicombustível (combinação de gás e diesel). Estas tecnologias estão reduzindo a vantagem histórica do custo dos geradores a gás contra os geradores a diesel. A frequência da tensão de saída do gerador é uma função do desenho da velocidade do motor e alternador. Para atingir $60 \mathrm{~Hz}$, o rotor do alternador deve operar a uma velocidade específica para uma determinada configuração dos polos do alternador. Cinquenta anos atrás, a maioria dos motores de geração operavam em velocidades abaixo de 900 rpm. Nos últimos 30 anos, no entanto, a velocidade do motor foi aumentada. Assim, a velocidade dos motores para geração foi alterada para 1200 a 1800 rpm. Esta tendência tem afetado os geradores a gás em aplicações de até $150 \mathrm{kWh}$. Historicamente operado a $1800 \mathrm{rpm}$, a tecnologia atual otimiza os motores automotivos para operar em 2300, 3000 e 3600 rpm. Alguns fabricantes utilizam uma caixa de redução simples entre motor e alternador de quatro polos para obter o melhor rendimento mecânico / elétrico dos equipamentos (GENERAC Grupos Geradores). A figura 1 mostra o esquema básico de um gerador a gás. 


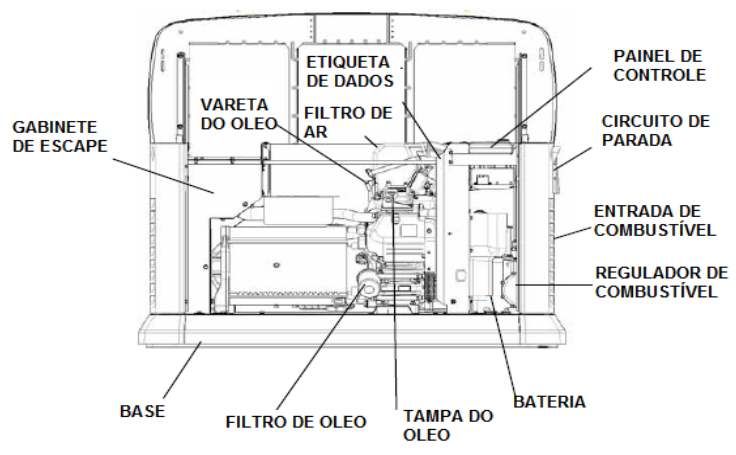

Figura 1 - Gerador a gás

\subsection{Combustíveis gasosos}

Em comparação aos combustíveis líquidos, os combustíveis gasosos possuem uma relação entre as moléculas de hidrogênio e carbono reduzida, desta forma a geração de $\mathrm{CO}_{2}$ na combustão é menor, tendo uma maior geração de vapor de água. Possuem também maior facilidade em se misturar com o ar, ocasionando misturas homogêneas com facilidade de queima (MARTINS).

\subsubsection{Gás Liquefeito de Petróleo (GLP)}

Gás obtido através do processamento do gás natural. Geralmente chamado de gás de cozinha, é basicamente uma mistura de propano e butano, porém pode conter outros hidrocarbonetos em baixa quantidade. A sua queima resulta em baixos níveis de emissões. Como o gás é incolor, é adicionado um composto a base de enxofre, assim o gás é perceptível ao olfato (Agência Nacional de Petróleo). Possui como característica apresentar-se na forma líquida quando submetido a pressões superiores a $4 \mathrm{kgf} / \mathrm{cm}^{2} \mathrm{e}$ inferiores a $15 \mathrm{kgf} / \mathrm{cm}^{2}$. Pode ser considerado estável, por esta razão é de fácil transporte e armazenamento (Liquigás). Segundo a Universidade Petrobras, o GLP, pode ser constituído de propano $\left(\mathrm{C}_{3} \mathrm{H}_{8}\right)$, propeno $\left(\mathrm{C}_{3} \mathrm{H}_{6}\right)$, isobutano $\left(\mathrm{C}_{4} \mathrm{H}_{10}\right)$, n-butano $\left(\mathrm{C}_{4} \mathrm{H}_{10}\right)$ e buteno $\left(\mathrm{C}_{4} \mathrm{H}_{8}\right) . \mathrm{O}$ propano tem poder calorífico inferior de $49.952 \mathrm{~kJ} / \mathrm{kg}$ e o butano de $49.255 \mathrm{~kJ} / \mathrm{kg}$. Quando comparado aos demais combustíveis, o GLP possui uma queima limpa, com baixos níveis de SOx, NOx (poluentes secundários os quais na atmosfera dão origem aos ácidos sulfúrico e nítrico respectivamente) e $\mathrm{CO}_{2}$. Segundo a resolução ANP № 18 de 02/09/2004, a classificação propano/buteno do GLP, pode conter percentuais variáveis de propano, propeno, butano e buteno, ficando a cargo do distribuidor a escolha da composição a ser vendida. O GLP pode ser comercializado em embalagens (botijões) que variam de 2 a 90 $\mathrm{kg}$, no estado líquido. Desta forma, seu uso se torna mais conveniente em residências e locais remotos (UNIVERSIDADE PETROBRAS). 


\subsubsection{Gás Natural Veicular (GNV)}

Combustível gasoso, produzido através do refino do gás natural ou biogás, com concentração predominante de metano. A composição do gás natural encontrado na forma bruta pode variar muito, porém, o metano predomina a composição acompanhado de etano, propano e butano (LORA, NASCIMENTO). A extração ocorre diretamente nos reservatórios petrolíferos, havendo grande variação na proporção de gás de um reservatório para outro. Em alguns reservatórios o gás natural está associado ao óleo, as vezes dissolvido no próprio óleo ou apenas em contato. Nestas condições o gás natural acaba se tornando apenas um subproduto do petróleo, onde seu aproveitamento é economicamente inviável. Em outros reservatórios, o gás natural não está associado ao óleo, é predominante no reservatório e em alguns casos único. Nestes casos há viabilidade econômica para produção do gás, sendo predominante esta forma de produção no mundo. Para alcançar as propriedades desejadas para comercialização, o gás natural bruto passa por um tratamento em uma Unidade de Processamento de Gás Natural (UPGN), que realiza a retirada de impurezas e de hidrocarbonetos pesados. O gás natural comercializado é constituído basicamente por metano, e as quantidades de etano e propano são somente suficientes para elevar o poder calorífico inferior ao valor desejado. Desta maneira, as análises termodinâmicas podem ser efetuadas levando em consideração o metano como único gás presente na mistura. Com esta consideração é possível assumir que o gás natural possui como poder calorífico inferior 49.000 kJ/kg (GÁS Natural).

\subsection{Redutor, misturador e ignição}

O redutor de pressão tem por finalidade adequar a pressão do gás que se encontra no reservatório com aquela admitida para combustão (ACIOLI). Para o GNV a pressão de armazenamento é de 220 bar e é necessário a utilização de um redutor específico, com capacidade de disponibilizar uma pressão de saída de aproximadamente 1,5 bar. O GLP é armazenado a pressões inferiores ( 7 bar) em comparação ao GNV, desta forma o redutor de pressão possui características diferentes.

O misturador, também chamado de mesclador, tem como função principal realizar a correta mistura entre o ar que está sendo admitido pelo motor e o gás que está sendo usado como combustível (CASTRO). Este evento se dá de forma correta em função do tipo de escoamento e da geometria da peça. A capacidade de mistura pode ser incrementada através dos vórtices gerados por um escoamento turbulento.

Os combustíveis gasosos, com exceção do H2, possuem velocidade de propagação de chama reduzida em comparação aos combustíveis líquidos, desta forma é necessário um sistema de ignição mais robusto, com maior capacidade de descarga de energia (MARTINS). 


\section{I MATERIAIS E MÉTODOS}

\subsection{Testes do gerador a gás}

O gerador a gás de 5,5 kVA é o grupo motor-gerador SP6500EB-F a GLP da empresa S\&S Grupo Geradores, descrito no item 1.2. Devido a questões de segurança, primeiramente foi enviado aos Laboratórios da Automotiva da Fundação Escola Técnica Liberato Salzano Vieira da Cunha para testes preliminares com GLP e GNV de acordo com as normas ANP Nº 685 de 29/06/2017 e ANP N ${ }^{\circ} 16$ de 17/06/2008. Estas normas estabelecem que o biometano a partir de biogás deve ter a mesma formulação do GNV. Assim, os testes com GNV são plenamente válidos para o biogás a partir de biometano.

A equipe deste projeto constatou que o gerador a gás adquirido é uma adaptação de um motor à gasolina da Shanghai Amazonas para gás GLP, acoplado a um gerador elétrico monofásico. Esta adaptação é realizada pela própria empresa que fornece este equipamento.

O gerador utilizado nos testes foi adquirido pronto para o uso com combustível gasoso. Para a utilização de GLP como combustível, é necessário realizar a conexão entre o reservatório de gás e o redutor de pressão presente no gerador originalmente. Ao utilizar o reservatório de GNV com 220 bar foi necessária a instalação de um redutor externo. A figura 2-a mostra o redutor utilizado para o GNV e a figura 2-b para o GLP.

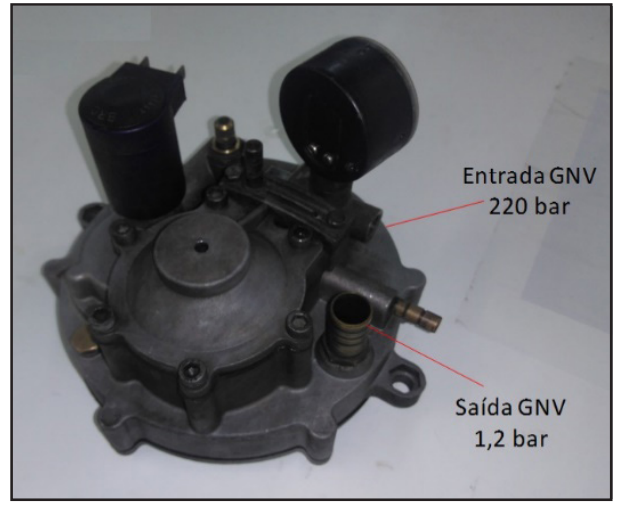

(a)

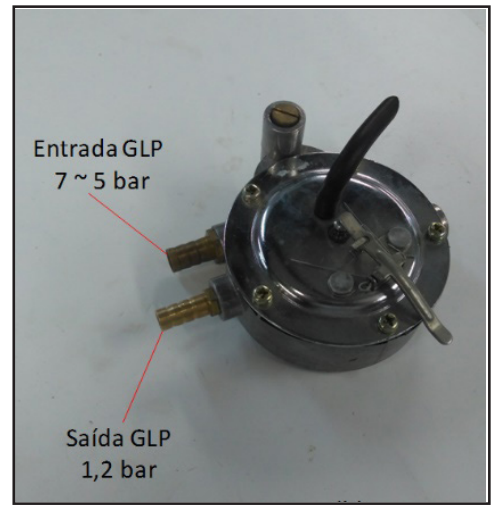

(b)

Figura 2 - Redutores de pressão para (a) GNV e (b) GLP

O redutor de pressão para GNV é utilizado em veículos com motores de até $100 \mathrm{~kW}$, fabricado pela empresa BRC modelo ME. Este redutor possui três estágios de membranas e regulagem automática do fluxo de gás, conforme as solicitações do motor. Já o redutor para o GLP é de constituição mais simples pois é utilizado para uma pequena redução. Como o gerador adquirido está apto para uso com combustíveis gasosos, o sistema de 
ignição não foi alterado. O gerador utilizado nos testes teve o sistema de atomização de combustíveis líquidos desativado, sendo este, adaptado como um misturador para combustíveis gasosos.

Durante os testes, o misturador não se mostrou eficaz. O gerador só alcançou um funcionamento linear quando aplicada uma restrição na admissão do motor. Assim, nestes testes preliminares, foi verificado que este gerador funciona perfeitamente com GLP, GNV e, em consequência, com biometano.

Encerrados os testes preliminares, o gerador a gás foi enviado para a Oficina Tecnológica da Engenharia Mecânica da Universidade Feevale, onde foram realizados os testes definitivos a base de GLP e GNV, a fim de comparar o desempenho a partir dos destes dois combustíveis. Em razão de o gerador a gás tratar-se de um grupo motorgerador, ou seja, um motor a combustão que aciona um gerador elétrico, os testes de potência devem ser realizados colocando-se uma carga resistiva na saída do gerador. Para este teste foi utilizado um banco de carga resistiva de 7,5 kW.

O primeiro teste foi realizado utilizando GLP e consistiu da pesagem de um botijão de gás (figura 3-a), antes e após um determinado tempo de funcionamento, alimentando o banco de carga resistiva (figura3-b). Assim, a partir da pesagem do gás consumido e da potência dissipada na carga resistiva observadas a partir dos medidores de tensão e corrente (figura 3-c), foi possível relacionar a quantidade de gás consumida pelo motor a combustão e a potência gerada na saída do gerador. O teste com GNV seguiu a mesma metodologia.

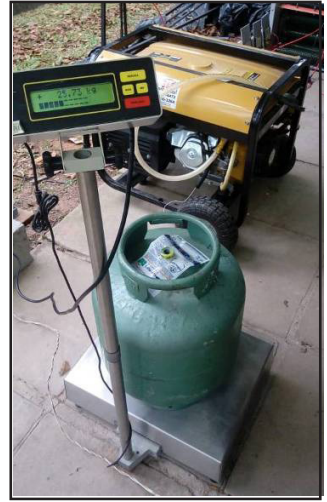

(a)

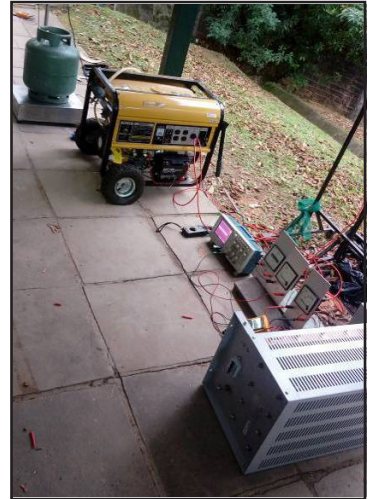

(b)

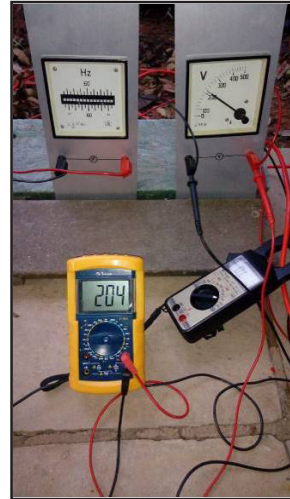

(c)

Figura 3 - Testes definitivos do gerador a gás com GLP - (a) gás sendo pesado - (b) gerador conectado a carga resistiva e (c) instrumentos de medição 


\subsection{Instrumentos de medição}

A instrumentação do gerador a gás teve como objetivo adicionar sistemas de medições para uma melhor observação do que estava acontecendo. Foram acrescentados ao gerador a gás medidores de temperatura, tensão e corrente elétrica. Além destes, foram utilizados uma balança digital e um banco de cargas resistivas.

Para uma melhor aferição da temperatura do gerador, além do termopar da admissão, foi também instalado outro termopar na saída dos gases de escape do motor, para medir a temperatura dos gases de escape. O mesmo termômetro foi usado para aferir ambas as temperaturas, apenas foi alternado o termopar conectado.

Nos testes para as medidas de tensão, foram utilizados multímetros da marca MINIPA, com escalas para $220 \mathrm{~V}_{\mathrm{rms}}$. Para os testes de medida de corrente foi utilizado um amperímetro de alicate com capacidade para $200 \mathrm{~A}_{\mathrm{AC}}$ da marca MINIPA. A visualização das formas de onda e outros dados de medição de tensões elétrica foram realizados utilizado um osciloscópio digital TECTRONIX.

Para as medidas de massas dos cilindros dos gases foi utilizada uma balança da marca TOLEDO, com escala para até $150 \mathrm{~kg}$ (figura 6-a).

Os testes de consumo do gerador foram realizados utilizado um banco de carga resistivo de $7,5 \mathrm{~kW}$ para tensão de $220 \mathrm{~V}_{\mathrm{AC}}$ fabricado por OHMIC, com 9 resistências de potência nominal de $866 \mathrm{~W}$ e máxima de $1 \mathrm{~kW}$ cada, o que totaliza, dependendo do tipo de ligação, em uma potência máxima de $9 \mathrm{~kW}$ (este é um valor limite, somente podendo ser atingido por curtos períodos de tempo para tensões maiores que $220 \mathrm{~V}_{\mathrm{AC}}$ ). Para facilitar as ligações dos cabos, foram adicionados conectores externos ao banco de carga, possibilitando assim, maior agilidade na mudança da carga.

\subsection{Metodologia dos testes}

Os testes de operação do grupo motor-gerador para os gases combustíveis GLP e GNV foram realizados variando-se a carga ligada na saída monofásica do gerador. 0 primeiro teste foi executado com carga de $2,5 \mathrm{~kW}$ e o segundo com carga de $4,5 \mathrm{~kW}$, ambos ligados conforme mostra o diagrama em blocos da figura 4.

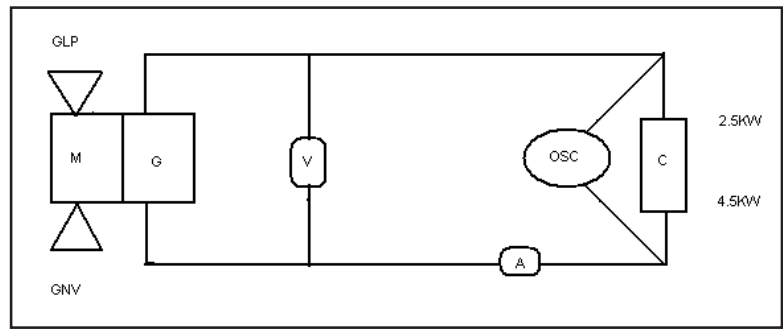

Figura 4 - Diagrama em blocos do Sistema de Medição utilizado nos testes 


\section{I RESULTADOS E DISCUSSÕES}

Os resultados encontrados no primeiro teste para uma carga na saída de $2,5 \mathrm{~kW}$ mostraram uma relação entre o consumo de gás, potência gerada e tempo, resultou em um consumo de GNV 57\% menor que de GLP. Esta relação comprova o bom desempenho do GNV e biometano. Cita-se que o biometano é um gás obtido a partir de dejetos e resíduos orgânicos, portanto uma fonte de energia renovável.

O segundo teste, tanto com GLP quanto com GNV, foi realizado considerando uma carga resistiva com potência máxima de $5 \mathrm{~kW}$ e observou-se que com o aumento da potência na carga resistiva, a frequência da tensão gerada, que era maior que $60 \mathrm{~Hz}$ no primeiro teste, caiu para $45 \mathrm{~Hz}$ para o GLP e $44 \mathrm{~Hz}$ para o GNV. Tal condição fez com que fosse limitada a potência do gerador a gás em $3 \mathrm{~kW}$, e não mais os $5,5 \mathrm{~kW}$ indicados pelo fabricante do gerador. A tabela 2 mostra os resultados dos testes.

\begin{tabular}{|c|c|c|c|c|}
\hline \multirow{2}{*}{ PARÂMETROS } & \multicolumn{2}{|c|}{ GLP } & \multicolumn{2}{c|}{ GNV } \\
\hline Avaliação & $\begin{array}{c}\text { Meia } \\
\text { carga }\end{array}$ & Carga & Meia carga & Carga \\
\hline Duração do teste [min] & 15 & 15 & 30 & 30 \\
\hline Resistor de carga [ $\Omega$ ] & 19,7 & 9,9 & 19,7 & 9,9 \\
\hline Tensão [VAC] & $218+/-1$ & $215+/-1$ & $219+/-0,1$ & $204+/-2$ \\
\hline Corrente [A] & 11,3 & 21,9 & 10,7 & 19,7 \\
\hline Frequência [Hz] & 61.7 & 45 & 63 & 44 \\
\hline$\Delta \mathrm{F}(+/-)$ & $+/-0,5$ & $+/-2$ & $+/-0,4$ & $+/-2$ \\
\hline Consumo [g] & 750 & 600 & 650 & 850 \\
\hline Potência [kW] & 2.46 & 4.708 & 2.33 & 4.05 \\
\hline Temperatura carcaça [ $\left.{ }^{\circ} \mathrm{C}\right]$ & 60 & 71 & 65 & 65 \\
\hline
\end{tabular}

Tabela 2 - Resultados dos testes

Os valores de tempo de testes foram diferentes devido ao baixo consumo em GNV. Mas as análises foram proporcionais aos consumos com os 2 gases.

A carga apresenta condição de ligação em série ou paralelo das resistências para se alcançar a potência desejada.

A tensão de saída com meia carga o gerador se comportou estável, mantendo a 
tensão e frequência nominais até $2,5 \mathrm{~kW}$. Mas com carga plena, cerca de 4,5 kW, não houve estabilidade de tensão e frequência, identificando necessidade de melhor adaptação do sistema de válvulas de admissão dos gases. Após 5 minutos de teste com CARGA PLENA, a frequência caiu para $45 \mathrm{~Hz}$ em função de sobrecarga no motor nos dois tipos de gás.

A eficiência do sistema apresentou-se válida para cargas de até 2,5 kW, mostrandose eficaz para a transformação de energia nestes parâmetros. Contudo, mesmo dentro da faixa de potência de construção do grupo motor-gerador, utilizando gases mostra-se ineficiente para cargas maiores.

Para a relação custo x peso, observou-se que o preço médio do GNV foi de $R \$$ $2,899 / \mathrm{m}^{3}$ e do GLP foi de $\mathrm{R} \$ 13,076 / \mathrm{m}^{3}$, sendo que o teste indicou um consumo com menor custo utilizando GNV.

\section{I CONCLUSÕES}

Analisando-se os testes com uso de gases como combustível, conclui-se que esse tipo de motor apresenta um rendimento abaixo do esperado, pois manteve-se estável apenas até 2,5 kW. Acima deste valor a frequência e tensão caíram na saída do gerador. Uma forma de solucionar este problema seria acoplar um motor à combustão de maior potência.

Diante das dificuldades encontradas no decorrer do trabalho, constatou-se a necessidade de aprofundar a pesquisa das válvulas de alimentação dos gases, pois aquelas utilizadas nos testes apresentaram falhas de regulagem e vazão. Estas falhas resultaram em sinais de congelamento nas válvulas de rebaixamento de pressão do GNV.

A análise financeira dos combustíveis empregados mostrou que embora exista diferença de preço do metro cúbico e grande diferença das relações massa-volume-pressão entre os dois gases, a utilização do GNV apresenta um melhor custo-benefício.

\section{REFERÊNCIAS}

ACIOLI, Rejane. Fabricantes de Redutores de Pressão garantem resistência acima dos

limites. 2006. Disponível em: http://www.gasnet.com.br/conteudo/26

51/Fabricantes-de-Redutores-de-Pressao-garantem-resistencia-acima-dos-limites. Acesso em: 10 de jun. de 2018.

Agência Nacional de Petróleo - Gás Liquefeito de Petróleo: GLP. 2017. Disponível em: http://www. anp.gov.br/petroleo-e-derivados2/glp. Acesso em: 28 de abr. de 2018.

CASTRO, Jonathas Assunção S.n. de. Otimização de um Misturador Ar-GNV. 2008. 91 f. TCC

(Graduação) - Curso de Engenharia Mecânica, Universidade de Brasília, Brasília, 2008. 
GÁS Natural. 2013. Disponível em: http://www.gasnet.com.br/gasnatural/gas_co mpleto.asp\#gas1. Acesso em: 29 de abr. de 2018.

GENERAC Grupos Geradores. Disponível em: http://www.generacbrasil.com.b r/galeria/geradores-de-energia-a-gasl>. Acesso em: 01 de set. de 2019.

Grupo GMEG. Disponível em: http://grupogmeg.com.br/produtos/motomil/?gclid =Cj0KCQiAtvPjBRDPARIsAJfZzOrzfgUpclVdad2OAselONoy3tEBVulyD3_vjafan5jh0tU_ EyaQiygaAjmSEALw_wcB. Acesso em: 01 de set de 2019.

Liquigas - O que é GLP? Disponível em: www.liquigas.com.br. Acesso em: 28 de abr. de 2018.

Lora, E.E.D.S.; Nascimento M.A.R.D. Geração Termelétrica - Planejamento, Projeto e Operação, 2 ed., EDITORA, CIDADE, 2004

MARTINS, Jorge. Motores de Combustão Interna: Quarta Edição, Revista e aumentada. 4. ed. Porto: Publindústria, 2013. 473 p.

Stemac grupo geradores. Disponível em: http://www.stemac.com.br/pt/produto s/Pages/grupos-geradores-gas-natural.aspx. Acesso em: 01 de set. de 2019.

S\&S Grupos geradores. Disponível em: https://www.lojassgeradores.com.br/ge rador. Acesso em: 01 de set. de 2019.

Toyama. Disponível em: http://www.toyama.com.br. Acesso em: 01 de set. de 2019.

UNIVERSIDADE PETROBRAS. Escola de Ciências e Tecnologias de Abastecimento. Gás Liquefeito de Petróleo: Informações Técnicas. Rio de Janeiro: Comissão de Assistência Técnica Petrobras, 2013. $11 \mathrm{p}$. 


\section{ÍNDICE REMISSIVO}

\section{A}

Acabamento 2, 3, 9, 10, 11, 15, 16, 17, 22, 25, 28, 75, 151, 152

Aço Carbono 22, 25

Aço N2711 8, 13

Algoritmos 128, 129, 131

Aplicações Matemáticas 128

Atmosfera de Corte 19, 151, 152, 154, 155, 156, 157

B

Biodegradável 49, 52

Biomodelos 71, 73

Blindagem 31, 32, 33, 34, 35, 36, 42, 45, 46

C

Caracterização Mecânica 47, 48

Carbeto de Silício 15, 16, 17, 18, 19, 20

Circuitos Shunt Piezelétricos 81

Coeficiente Convectivo 104, 106, 110

Coletor Solar 117, 126

Controle Ótimo 91, 128, 129, 130, 135, 138

Controle Passivo 81, 82, 86, 91

Correlações 104, 105, 106, 107, 108, 109, 110, 111, 112, 113, 114

D

Distância de Aplicação 22, 24, 25, 29

E

Elementos Finitos 71, 74, 76, 77, 79, 80, 81, 82, 84, 85, 92

Embalagens 48, 52, 96

Energia Renovável 94, 101

Escoamento Multifásico 104

G

Gás Liquefeito de Petróleo 94, 96, 102, 103

Gás Natural Veicular 94, 95, 97

Grupo Motor-Gerador 93, 94, 95, 98, 99, 100, 102 
Imagem Médica 71

Inconel 718 15, 16, 17, 18, 19, 20, 21

Integridade da Superfície 8

J

Jateamento 22, 23, 24, 25, 26, 27, 28, 29, 30

L

Laminados de Aço 31, 32, 36, 39

M

Materiais Absorvedores 60, 61, 70

Material Compósito 81, 82, 85, 86

Modelagem 71, 72, 76, 79, 81, 82, 83, 84, 86, 92, 104, 138, 141, 143

Modelo Teórico 1, 2

Módulos Balísticos 31

N

Nanopartículas 61

$\mathbf{P}$

Padrão Intermitente 104, 105, 107, 108

Penetração de Trabalho 2, 3, 4, 5, 8, 9, 10, 11, 12, 13, 15, 17, 18, 19, 20, 151, 152, 153, 154, 155, 156, 157

Potência Elétrica 151, 152, 153, 154, 155, 156, 157

Propagação de Incertezas 81

Próteses 71,73

$\mathbf{R}$

Radiação Térmica 117, 118, 119, 122, 123, 126

Refletividade 60, 61, 122, 123, 124, 126

Retificação 1, 2, 3, 4, 5, 6, 8, 9, 10, 11, 12, 13, 14, 15, 16, 17, 18, 19, 20, 21, 28, 29, 151 , 152, 153, 154, 155, 156, 157

Rugosidade $1,2,3,4,5,6,8,9,10,11,13,15,17,18,19,20,22,25,27,28,29,30,151$

S

Superfícies Seletivas 117, 118, 127 
Teodolito 139, 140, 141, 143, 145, 146, 147, 148, 149, 150

Transferência de Calor 104, 105, 106, 107, 108, 109, 110, 113, 115, 118, 126, 127

V

Velocidade da Peça 2, 3, 4, 5, 8, 10, 12, 17, 18, 151, 152, 153, 154, 155, 156, 157 
(1) www.atenaeditora.com.br

contato@atenaeditora.com.br

이@atenaeditora

f www.facebook.com/atenaeditora.com.br

\section{A Aplicação do Conhecimento Científico na Engenharia Mecânica}
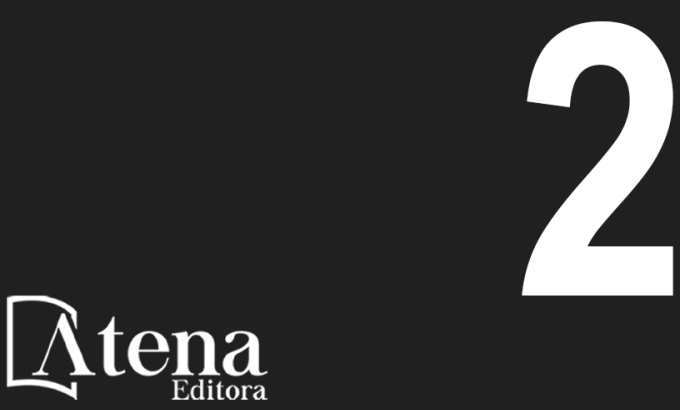

Ano 2020 
(1) www.atenaeditora.com.br

$\triangle$ contato@atenaeditora.com.br

(0)@atenaeditora

f www.facebook.com/atenaeditora.com.br

\section{A Aplicação do Conhecimento Científico na Engenharia Mecânica}
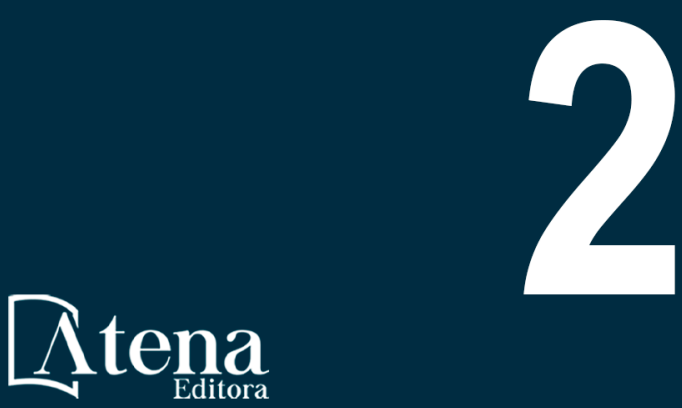

Ano 2020 